\title{
Quality of Life and Psychiatric Morbidity in Caregiver of Alcohol Dependence Patients
}

\author{
Jaydeepsinh G. Gohil ${ }^{1}$, Mukesh K. Patel ${ }^{2}$, Mukesh J. Samani ${ }^{3}$ \\ 1,2,3 Department Of Psychiatry, PDU Medical College, Rajkot, Gujarat, India
}

\begin{abstract}
Alcohol dependence affects not only patient but its deleterious effects are extended to family members. They are most vulnerable to have significant psychiatric disorders.

Aims: to investigate the quality of life and the frequency and nature of psychiatric morbidity in caregiver of patients with alcohol dependence.

Material and method: The study was carried out at Psychiatry OPD, P.D.U. Medical College \& Hospital, Rajkot. In this cross-sectional study, 110 patients between ages 18-65, having alcohol dependence as per diagnostic criteria of DSM - V was studied. One caregiver of each patient was also studied. Leeds dependence questionnaire was administered in patients. GHQ and Q-LES-Q-SF Scales were administered in caregivers. The results were tabulated and analyzed using chi $(\chi 2)$ square test and Mann Whitney U test. Correlation coefficient was measured between scores of all scales using Pearson's formula.

Results: Quality of life of was found to be significantly poor among caregivers of alcohol dependent patients. Out of 110 caregivers, 40(36\%) caregivers had dysthymia, 24(22\%) had major depression, 9(8\%) had unspecified anxiety disorder and 5(4.5\%) had generalized anxiety disorder.
\end{abstract}

Conclusion: The Study suggests need for sensitization of psychiatrists to evaluate and treat caregivers of alcohol dependent patients.

Keywords: alcohol dependence, caregiver, caregiver burden, psychiatric morbidity, quality of life.

\section{Introduction}

The caregiver of patients with alcohol use disorders are on most occasions the core members of the dysfunctional family system. Problems such as family conflicts and poverty caused by patient's alcohol use can be the main cause of caregivers' suffering and can result in the caregiver losing meaning in their lives and experiencing despair and hopelessness. They are quite vulnerable to have significant psychiatric disorders like mood disorders, anxiety disorders and a higher prevalence of medical morbidity and psychosocial problems as well. However, the psychiatric morbidity in the caregiver of patients with alcohol dependence is a largely neglected area in psychiatric research. We come across only a few published papers about psychiatric disorders in caregivers of patients with alcohol dependence.

\section{Materials And Methods}

This is a cross- sectional study involving all the consecutive indoor and OPD patients with alcohol use disorder in Department of Psychiatry, PDU Medical College, Rajkot (which is a tertiary care centre) and their caregivers were taken for study. The study was done during the period from April 2014 to March 2015. Patients were clinically evaluated in detail with history, mental status examination and diagnosed by using DSM-5 criteria.

They were explained about the nature of the study and informed written consent was taken). Screening of caregivers was done by using GHQ and GHQ positives were evaluated for psychiatric morbidity using DSM5 criteria. ${ }^{[1]}$ Quality of life of caregivers was determined using Quality of Life Enjoyment and Satisfaction Questionnaire - Short Form (Q-LES-Q-SF). Severity of alcohol dependence was assessed by using LEED'S Dependence Questionnaire.

SPSS version 17.0 was used to analyze data. Data was analyzed for statistical significance with chi square test and t-test as the case may be. Probability value less than 0.05 has been taken as statistically significant. Pearson correlation was used to find out correlation between quality of life and severity of illness and psychiatric morbidity. Correlation is considered significant at the 0.01 level (2-tailed). Result is presented and discussed in reference to previous studies.

\section{Leed's Dependence Questionnaire}

Leeds dependence questionnaire was used to assess the severity of alcohol dependence. It contains 10 questions to assess the severity of alcohol dependence. All items are scored 0-1-2-3; there are no normative data. A principal components analysis produced a single factor accounting for $64 \%$ of the variance. Cronbach's alpha was 0.94 . Test-retest reliability was found to be 0.95 . 


\section{Quality of Life Enjoyment and Satisfaction Questionnaire Short Form (Q-LES-Q-SF)}

The Q-LES-Q was developed in the USA in 1993 by Endicott et al for use in depressive patients to assess respondents' degree of enjoyment and satisfaction in different areas of daily life. It is a self-administered instrument. The long version of the Q-LES-Q includes 60 items with 33 additional optional items. The Q-LESQ-SF, which is identical to the General Activities subscale of the longer version of the Q-LES-Q instrument, includes 16 items. Each item is scored on a 5-point scale from "very poor" to "very good". The global QoL index can be summed of the first 14 items. Two last items deal with medication and overall life satisfaction. It takes about five minutes to complete the Q-LES-Q-SF scale. The various items in the instrument involve health, mood, work, relationship, etc. The Q-LES-Q-SF has established internal consistency, test-retest reliability.

\section{General Health Questionnaire (GHQ-12)}

The General Health Questionnaire (GHQ) is a measure of current mental health and since its development by Goldberg in the 1970s; it has been extensively used in different settings and different cultures. The questionnaire was originally developed as a 60 -item instrument but at present a range of shortened versions of the questionnaire including the GHQ-30, the GHQ-28, the GHQ-20, and the GHQ-12 is available. The scale asks whether the respondent has experienced a particular symptom or behavior recently. Each item is rated on a four-point scale (less than usual, no more than usual, rather more than usual, or much more than usual); and for example when using the GHQ-12 it gives a total score of 36 or 12 based on the selected scoring methods. The most common scoring method is bi-modal (0-0-1-1). GHQ-12 is a quick, reliable and sensitive short form which is ideal for research studies.

\section{Results}

Out of 110 caregivers, $78(71 \%)$ suffered from one or another DSM-V diagnosis suggesting very high impact of alcohol dependence of patient on their caregivers. 40(36.4\%) of caregivers had dysthymia, 24(21.8\%) had major depressive disorder, $9(8.2 \%)$ had other specified anxiety disorder and $5(4.5 \%)$ had generalized anxiety disorder, 32(29\%) had no psychiatric morbidity.

There was statistically significant difference between Q-LES-Q-SF score of caregivers who had psychiatric morbidity and caregivers who had no psychiatric morbidity $(\mathrm{z}=-6.821, \mathrm{p}=0.000)$. Statistically significant difference was also found between GHQ score of caregivers who had psychiatric morbidity and caregivers who had no psychiatric morbidity $(\mathrm{z}=-8.136, \mathrm{p}=0.000)$. No significant correlation was found between age, relation with patient, occupation, education, socioeconomic class of caregivers and psychiatric morbidity.

Severity of alcohol dependence as decided by LEED's Dependence Score was not a significant factor which can influence caregiver's quality of life as decided by Q-LES-Q-SF Score $(\mathrm{z}=-1.945, \mathrm{p}=0.052)$. "p" value 0.052 suggests some trends towards significance but because of smaller sample size it does not reach to significance value.

\section{Discussion}

\section{Psychiatric Morbidity In Caregiver Of Patients With Alcohol Dependence}

In our study, we had found significant psychiatric morbidity in caregiver of alcohol dependent patients. We found $36 \%$ (40) caregivers were having dysthymia, 22\% (24) caregivers were having major depressive disorder, $8 \%$ (9) caregivers were having unspecified anxiety disorder and 4.5\% (5) caregivers were having generalized anxiety disorder. Out of 110 caregiver of alcohol dependent patient, 78 (70.9\%) caregivers had psychiatric morbidity that is very high. Similar study by Caroline Dias (2013) ${ }^{[2]}$ showed that psychiatric disorders diagnosed in $75 \%$ of the wives of patients with alcohol dependence syndrome out of which $45 \%$ have dysthymia, $12 \%$ have mild depressive episode, $8 \%$ have adjustment disorder- prolonged depressive reaction, $5 \%$ have moderate depressive episode and 5\% have anxiety disorder unspecified. Kishor, Lakshmi V. Pandit, R. Raguram (2013) ${ }^{[3]}$ found that more than half of the spouses $(65 \%)$ had a psychiatric disorder which is near similar to our study (70.9\%). Major depressive disorder was present in $43 \%$ in spouses of alcohol dependent patients which is almost double than our study. Fulkunishi (1992) ${ }^{[4]}$ reported prevalence of alexythymia was $47.9 \%$ and that of depression was $63.3 \%$. In our study, caregivers of alcohol dependent patient had major depression prevalence of $22 \%$ which is not similar to the above study. Tomelleri (1978) ${ }^{[5]}$ found that psychiatric diagnosis in caregiver of alcohol use disorder in $15 \%$, hysteria in $12 \%$ and primary affective disorders in $8 \%$ of the wives.

The present study has not found a significant association between the duration and severity of alcohol dependence and the total psychiatric morbidity among the caregivers of patients with alcohol dependence. In study by Steinglass (1981) ${ }^{[6]}$ found that the psychopathology in the spouse is possibly proportional to the degree of alcohol dependence and with the husband's social impairment. This result is not similar to our study. 


\section{Impact Of Alcohol Dependence On Quality Of Life And Family Burden}

Our study had found that the quality of life was found to be poor in caregiver who had psychiatric morbidity as compared to that caregiver who had no psychiatric morbidity. Because of patient's alcohol drinking habit, it had affected various aspect of life of caregiver like economic, psychological, social, health, interpersonal, etc. We have found that as the age of the caregiver increases, the quality of life decreases. Caroline Dias (2013) ${ }^{[2]}$ found that the quality of marital life among wives of patients with alcohol dependence syndrome is poorer when compared to wives of patients with bipolar affective disorder. Samira Reschetti Marcon (2012) ${ }^{[7]}$ found that significant correlation between quality of life, depression and stress of caregivers. Findings confirmed that quality of life is compromised and stress is high among caregivers.

Quality of life of caregivers may improve with abstinence from alcohol by patients. Shruti Srivastava and Manjeet S. Bhatia (2013) ${ }^{[8]}$ concluded that significant improvement in quality of life in caregivers of patients of alcohol dependence over three months' abstinence.

We had not applied any scale to assess burden of caregiver of alcohol dependence patient, however as per our study we found significant number of caregiver $(71 \%)$ were having psychiatric illness and hence we can comment that a moderate to severe burden on caregiver of alcohol dependence patients and that burden may act like a mediator and may be responsible for psychiatric illness in caregivers. Similar study done by Prerna Malik $\mathrm{Et} \mathrm{Al}(2012)^{[9]}$ found that majority of primary caretaker (77.5 percent) was found to have moderate burden especially in financial areas, disruption of routine activities, family leisure and family interaction. Vijayanath $\mathrm{V}$. and Anitha M.R. (2013) ${ }^{[10]}$ found that burden experienced by caregivers of the patients having severe dependence was significantly more than the caregivers of low and moderate alcohol dependence group.

\section{Role Of Socio-Demographic Factors In Psychiatry Morbidity}

In the present study, the total psychiatric morbidity has been compared with the various socio demographic and clinical variables to find out the relationship if any between them. We have found significant correlation in sex and marital status of caregiver who had psychiatric morbidity and who had not.

We also found that most of the caregivers who had psychiatric morbidity were housewives and female. So we can't comment on the relation between occupation (housewife) and sex of caregiver and psychiatric morbidity. To overcome this problem, we need to take larger sample size with caregiver who is not only housewife and not of female sex.

In our study we had not found any significant correlation between age, relation with the patient, domicile, socioeconomic class, education, occupation of caregiver of alcohol dependent patients. Similar results were obtained by Surendra Kumar Mattoo Et Al (2012), ${ }^{[11]}$ they found that it was associated neither with age, education or duration of dependence of the patients, nor with family size, type of caregiver or caregiver's education and occupation.

Amol Desai, Mahadeo Shinde, Vaishali Mohite (2014) ${ }^{[12]}$ concluded that most of the caregivers of alcohol dependent patients had less knowledge about mental illness. So, there is need to improve the knowledge about mental illness among caregivers of alcoholics. In our study, majority of caregivers of alcohol dependent patients were unaware of their mental illness and came to know about it when they attended their alcohol dependent patients for treatment purpose. Though we had not studied about this aspect in our study, our observation was found to be similar with above study.

\section{Conclusion}

Our study suggests that majority of caregivers (71\%) of alcohol dependent patients were suffering from psychiatric illness. This indicates need for sensitization of psychiatrists about this very important aspect of overall management of alcohol dependent patients as well as caregivers too. Therefore we must not neglect to evaluate caregivers of alcohol dependent patients for the presence of psychiatric symptoms.

Presence of alcohol dependence in patient is an important factor which influences caregiver's psychiatric morbidity and quality of life however, severity of alcohol dependence in patients was not significantly correlated with psychiatric morbidity in caregivers. So even low alcohol dependence also leads to psychiatric illness in caregiver of alcohol dependent patients.

Quality of life of was found to be significantly poor among caregivers with psychiatric illness than caregivers without psychiatric illness.

\section{Limitations}

The major limitation of this study is its cross-sectional nature. We have studied the prevalence and pattern of psychiatric morbidity and quality of life in caregiver of currently alcohol dependent patients. So we can't comment on the prevalence and pattern of psychiatric morbidity in caregivers of alcohol dependence patients who are in remission as well as who have ongoing dependence. 
There is no control group in our study. So we can't compare psychiatric morbidity and quality of life in caregiver of alcohol dependent patients with normal population.

We have conducted the study on the patients attending our hospital. These patients may not be representative of all the patients in the community, particularly those who have no access to mental health care. Also we have conducted the study only on 110 patients. A larger sample size is needed to comment more accurately on the prevalence and pattern of psychiatric morbidity and quality of life in caregiver of alcohol dependence patients.

QOL of caregiver of alcohol dependent patient may also depend on personality factor of caregiver which we have not taken into consideration in our study. However, our process of data collection we realize this and search for research articles of personality factors of caregiver. Majority of research were with very small sample size and not definitely conclusive, but suggestive of dependent, weak, passive aggressive, neuroticism and low self-esteem on different scales. Thus, result of our study might have been deviated because of personality factors of caregiver.

\section{Suggestion For Future Research}

To get a more accurate idea of the prevalence and pattern of psychiatric morbidity and quality of life in caregiver of alcohol dependent patient, large scale study which is community based, longitudinal and with some control group is needed.

\section{References}

[1]. Kaplan \& Sadock's, Synopsis of Psychiatry, 10 $10^{\text {th }}$ ed. Lippincott Williams \& Wilkins, 2007

[2]. Caroline Dias, Psychiatric disorders in wives of alcohol dependence syndrome, IJP-2013.

[3]. Kishor, Lakshmi V. Pandit, R. Raguram, Psychiatric morbidity and marital satisfaction among spouses of men with alcohol dependence, Indian J Psychiatry, 2013 Oct-Dec, 55(4): 360-365.

[4]. Fulkunishi, Alexithymia and depression in families with alcoholics; functioning; Clinical Psychology Review, 2003 December, 23(7): 959-997.

[5]. Tomelleri C J, Marijan Herjanic, Barbara L, Herjanic, Weztel R D, The wife of Alcoholics, Currents in Alcoholism,1978;1(4):2930 .

[6]. Steinglass $\mathrm{P}$, The impact of alcoholism on the family relationship between degree of alcoholism and psychiatric symptomatology, Journal of studies on alcohol,1981, 42:288-303.

[7]. Samira Reschetti Marcon et al., Quality of life and depressive symptoms among caregivers and drug dependent people, Rev Lat Am Enfermagem, 2012 Jan-Feb; 20(1):167-74

[8]. Shruti Srivastava and Manjeet S. Bhatia, Quality of life as an outcome measure in the treatment of alcohol dependence ( Industrial Psychiatry Journal, Jan-Jun 2013 | Vol. 22 | Issue 1).

[9]. Prerna malik et al., Impact of substance dependence on primary caretaker in rural Punjab, Delhi Psychiatry Journal, Vol 15, No. 1, April 2012.

[10]. Vijayanath V. and Anitha M.R., Outcome of alcohol dependence, disability and burden on caregivers in society, JPSI 2(1), Jan-Feb $2013,10-14$

[11]. Surendra Kumar Mattoo et al., Family burden with substance dependence: a study from India, Indian J Med Res 137, April 2013, pp 704-711.

[12]. Amol Desai, Mahadeo Shinde, Vaishali Mohite, Study about Knowledge of Mental Illness among Caregivers of Alcoholic's, International Journal of Science and Research (IJSR) ISSN (Online): 2319-7064. 